\title{
The Convergence of Galerkin and Collocation Methods with Splines for Pseudodifferential Equatious on Closed Curves
}

\author{
G. SCHMJ.DT
}

In der vorliegenden Arbeit wird die näherungsweise Lösung von Pseudodifferentialgleichungen iuf geschlossenen Kurven mittels Galerkin- und Kollokationsverfahren untersucht, die als Ansatzfunktionen polynomiale Splines benutzen. Es werden hinreichende und im allgemeinen notwendige Bedingungen für die Konvergenz dieser Verfahren in Sobolevräumen angegeben.

В преілагаемой работе рассматривается приблићениое решение исевдодифференциальных уравнений на замкитых нривых методами Галеркина и кольокацин, в которых прио̆линеное решение пщется в выде полиномиального сплайа. Даются достаточные и, как правило, также необходимые условия сходимости этих методов в пространствах Соболева.

'The present paper studies the approximate solution of pseudodifferential equations on closed curves using Galerkin and nodal collocation methods with polynomial splines. We give sufficient and in general necessary conditions for the convergence of these methods in Sobolev spaces.

\section{Introduction}

Various physical problems can be reduced to pseudodifferential equations on closed curves. These equations include for example linear differential equations, certain classes of first and second kind Fredholm integral equations, singular integral equations with Cauchy kernel and integrodifferential equations. There is a sizable literature on the numerical treatment of such equations.

It is the purpose of the present paper to investigate the spline approximation of elliptic pseudodifferential equations via standard Galerkin procedures and via nodal collocation methods. We shall give conditions which are sufficient and in special cases also necessary for the convergence of these methods in Sobolev noms and establish quasioptimal error estimates in a range of Sobolev spaces.

Let $T$ be a simple closed $C^{\infty}$-curve in the complex plane given by the equation $z=z(x), x \in[0,1]$. We identify functions $u(z)$ on $\Gamma$ with 1 -periodic functions $u(x)=u(z(x))$ on the real axis $\mathbf{R}$. Let $A$ be a classical pseudodifferential operator of order $2 n \in \mathbf{R}$ on $\Gamma$, whose complete symbol $a(x, \xi)(\xi \in \mathbf{R})$. has an asymptotic expansion

$$
a(x, \xi) \sim \sum_{i=0}^{\infty} a_{2 n-l}(x, \xi) \text { as }|\xi| \rightarrow \infty,
$$

where $a_{2 n-l}$ are $C^{\infty}$ for $\xi \neq 0$ and positively homogeneous of degree $2 n-l$ with respect to $\xi$. Throughout the paper $C^{\infty}$ denotes the set of all infinitely differentiable 1-periodic functions on $\mathbf{R}$ and $H^{s}$ the periodic Sobolev space of arbitrary real order $s$, i.e. the closure of $C^{\infty}$ with respect to the norm

$$
\|u\|_{s}:=\left\{\left|\hat{u}_{0}\right|^{2}+\sum_{0 \neq k \in Z}\left|\hat{u}_{k}\right|^{2}|2 \pi k|^{2 s}\right\}^{1 / 2},
$$


where $\hat{u}_{k}=\int_{0}^{1} u(x) \mathrm{e}^{-i_{\pi i k x}} d \dot{x}$. Note that the inner product in the Hilbert space $H^{8}$

$$
\langle u, v\rangle_{s}=\hat{u}_{0} \overline{\hat{v}}_{0}+\sum_{0 \neq k \in \mathbf{Z}} \hat{u}_{k} \overline{\hat{v}}_{k}|2 \pi k|^{2 s}
$$

extends to a duality between $H^{s+r}$ and $H^{s-r}$ for arbitrary $r \in \mathbf{R}$ and

$$
\sup _{v \in H^{t-r}} \frac{\langle u, v\rangle_{s}}{\|v\|_{s-r}}=\|\left. u_{i}^{i}\right|_{s+r} .
$$

Clearly, $\dot{H}^{s}$ is continuously and compactly imbedded in $H^{t}$ for $t<s$ and, moreover, imbedded in the space of 1 -periodic continuous functions $C$ for $s>1 / 2$.

The pseudodifferential operator $A$ with symbol (1.1) has the representation [1]

$$
A u(x)=\sum_{0 \neq k \in \mathbf{Z}} \hat{u}_{k} \dot{a}(x, 2 \pi k) \mathrm{e}^{2 \pi i k x}+\int_{0}^{1} K(x, y) u(y) d y
$$

where $u \in C^{\infty}$ and $K(x, y)$ is a smooth kernel. We note, that $A$ is called elliptic if its principal symbol $a_{2 n}(x, \xi) \neq 0$ on $\mathbf{R} \times\{ \pm 1\}$. Then it generates a bounded Fredholm operator in Sobolev spaces, $A \in L\left(H^{s+n}, H^{s-n}\right), s \in \mathbf{R}$, whose index is equal to the winding number $(2 \pi)^{-1}\left\{\arg a_{2 n}(x,-1) / a_{2 n}(x,+1)\right\}_{x \rightarrow 0}^{x+1}$. Our aim is to investigate the approximation of the equation

$$
A u=f
$$

by Galerkin and nodal collocation methods using polynomial splines.

Let $\Delta=\left\{x_{k}\right\}_{k=-\infty}$ be a 1 -periodic mesh of the real line, i.e. $x_{k}<x_{k+1}, x_{k+n}=x_{k}+1$ for some fixed $n \in \mathbf{N}$ and all $k \in \mathbf{Z}$. By $S_{d}(\Delta)$ we shall denote the space of all 1-periodic, $d-1$ times continuously differentiable splines of degree $d$ subordinate to the 'partition $A$. We have, $S_{d}(\Delta) \subset H^{s}$ if and only if $s<d+1 / 2$. The spline spaces' $S_{d}(\Delta)$ provide the important approximation property (cf. [6]]):

Let $h_{\Delta}=\max _{k}\left(x_{k+1}-x_{k}\right)$.'If $s<d+1 / 2$ and $s \leqq r \leqq d+i$ then for. any $u \in H^{r}$ and any partition $\Delta$ there exists $u_{\Delta} \in S_{d}(\Delta)$ such that

$$
\left\|u-u_{\Delta}\right\|_{t} \leqq c(t) h_{\Delta}^{r-t}\|u\|_{r}
$$

for all $t \leqq s, \bar{c}(t)$ denoting constants independent of $u$ and $\Delta$.

The standard Galerkin method for approximate solving equation (1.4) can be formulated as to find a spline $\dot{u}_{\Delta} \in S_{d}(\Delta)$ satisfying the Gálerkin equations

$$
\left\langle A u_{\Delta}, v_{\Delta}\right\rangle_{0}=\left\langle f, v_{\Delta}\right\rangle_{0} \text { for all } v_{A} \in S_{d}(\Delta) \text {. }
$$

'The nodal collocation of equation (1.4) reads as: Find $u_{\Delta} \in S_{d}(\Delta)$ such that' the collocation equations

$$
A u_{\Delta}\left(x_{k}\right)=f\left(x_{k}\right) \quad(k=1, \ldots, n)
$$

are satisfied.

We study the problem under which conditions on $A$, the right-hand side $f$ and the splines equations (1.6) resp. (1.7) are uniquely solvable for all sufficiently fine meshes $\Delta$ and the sequences of the approximate solutions $\left\{u_{\Delta}\right\}$ converge to a solution of (1.4) in certain Sobolev norms. 
There is a large literature on Galerkin methods and the following fact is well known (cf. $[3,14])$ :

Assume that $A$ is coercive elliptic, i.e.

$$
\operatorname{Re} a_{2 n}(x, \xi)>0 \text { on } \mathbf{R} \times\{ \pm 1\}
$$

and $\operatorname{dim} \operatorname{ker} A=0$. For $f \in H^{r-2 n}, n \leqq r \leqq d+1, n<d+1 / 2$, the Galerkin equations (1.6) are uniquely solvable for any mesh $\Delta$ with sufficienlly small $h_{\Delta}$ and the approximate solutions $u_{\Delta} \in S_{d}(\Delta)$ converge in the. norm of $H^{t}, 2 n-d-1 \leqq t \leqq n$, to the exact solution $u$ as $h_{\Delta} \rightarrow 0$ with optimal order $O\left(h_{\Delta}^{r-t}\right)$.

Obviously, this assertion. is valid if the principal symbol of $A$ satisfies $\operatorname{Re} \vartheta a_{2 n}(x, \xi)$ $>0$ for some.number $\vartheta \in \mathbf{C}$. In Section 4 we shall prove that the assertion holds if the pseudodifferential operator $A$ is strongly elliptic, i.e. there exists a functions $\vartheta \in C^{\infty}$ such that

$$
\operatorname{Re} \vartheta(x) a_{2 n}(x, \xi)>0 \text { on } \mathbf{R} \times\{ \pm 1\} .
$$

Moreover, we shall show that in a special case the strong ellipticity is even necessary for the convergence of Galerkin's method.

Although in practice most numerical computations for solving equation (1.4) employ collocation procedures, hitherto the convergence of these methods is rather completely studied only for certain special equations, as for Fredholm integral equations of the second kind and for ordinary differential equations. Recently it was shown in two papers that the nodal spline collocation converges for strongly elliptic operators. In [11] S. PrössDorf and G. Schmot state the $L^{2}$-convergence of the collocation with linear splines for a singular integral equations on the unit circle if and only if this equation is strongly elliptic. In [2] D. ARNOLD and W. WENDLAND developed a new and elegant technique to investigate the nodal collocation with splines of odd degree relating the collocation equations with certain nonstandard Galerkin equations. They proved the convergence of nodal collocation using splines of arbitrary odd degree $d>2 n$ for strongly elliptic pseudodifferential equations and obtained quasioptimal error bounds for any right-hand side $f \in H^{(d+1) / 2-x}$. Using these ideas and some facts about projection methods the author considered in [13] the convergence of the nodal collocation using splines of arbitrary degree $d \geqq 1$ for pseudodifferential operators of order zero, i.e. for singular integral operators

$$
a(z) u(z)+b(z) S u(z), \quad z \in \Gamma .
$$

Here

$$
S u(z)=\frac{1}{\pi i} \int_{\Gamma} \frac{u(\zeta)}{\zeta-z} d \zeta
$$

is the Cauchy singular operator having the symbol $\xi /|\xi|$.

In the present paper we generalize the results of [2] and [13] to pseudodifferential operators of arbitrary real order $2 n$ and for spline spaces $S_{d}(\Delta)$ of degree $d>2 n$. In order to formulate a special case of the results in Section 3 we define: A mesh $\triangle$ is said to be $\gamma$-quasiuniform $(\gamma>0)$ if min $\left(x_{k+1}-x_{k}\right) \geqq \gamma h_{\Delta}$. The set of all $\gamma$-quasiuniform meshes for some fixed $\gamma$ we shall denote by $\mathscr{D}_{\gamma}$. Then we can prove:

Let $\operatorname{dim} \operatorname{ker} A=0$ and $\gamma>0$ be fixed. For $f \in H^{r-2 n}, 1 / 2+2 n<r \leqq d+1$, the collocation equations (1.7) are uniquely solvable for any $\Delta \in \mathscr{D}_{\gamma}$ with sufficiently small $h_{\Delta}$ 
and the approximate solutions $u_{1} \in S_{d}(\Delta)$ converge in $H^{\prime}, 2 n \leqq t<d+1 / 2$ and $' t \leqq r$, to the exact solution $u$ with optimal order $\Theta\left(h_{d}^{r-t}\right)$ if $d$ is odd and the operator $A$ is strongly elliptic or if. $d$ is even and the operator $A S$ is strongly elliptic.

Note that in [13] we proved the necessity of these conditions for the convergence of the nodal collocation in special cases. :

Comparing these results we can state: The spline collocation converges for a wider class of pseudodifferential equations but for a sinaller set of right-hand sides than the standard Galerkin method. Moreover, the Galerkin procedure achieves higher rates of convergence.

The author is grateful to Professor W. Wenduaxd for bringing to attention the paper [2]. Also, he thanks Professor S. Prössdorf and Dr. J. ELschner for many hel pful discussions.

\section{Galerkin method in $\boldsymbol{H}^{\mathrm{s}}$}

In this section we collect some results on Galerkin procedures in Sobolev spaces $H^{s}$, which will be applied in Sections 3 and 4. As a rule the results can be easily deduced from standard literature $[3,15]$ and we shall only sketch the proofs.

Let $X_{h}(0<h<1)$ be a sequence of finitedimensional subspaces of $I^{s}$ for all $s<m \in \mathbf{R}$ having the approximation properiy

A 1: if $s<m$ and $s \leqq r \leqq m+1 / 2$ then for any $u \in H^{r}$ and any $h$ there exists $u_{h} \in X_{h}$ such that

$$
\left\|u-u_{h}\right\|_{t} \leqq c(t) h^{r-t}\|u\|_{r} \quad \text { for all } t \leqq s,
$$

$c(t)$ denoting constants independent of $u$ and $h$.

At some places we shall additionally require that the subspaces possess the inverse property

A 2: for $t \leqq s<m$ there exists a conslant $c$ such that

$$
\cdot\left\|v_{h}\right\|_{s} \leqq c h^{t-s}\left\|v_{h}\right\|_{\ell} \text { for all } h \text { and all } v_{h} \in X_{h} .
$$

The Galerkin method in $H^{\text {s }}$ for approximate solving equation (1.4) is defined is to find an element $u_{h} \in X_{h}$ satisfying the equation

$$
\left\langle A u_{h}^{\prime}, v_{h}\right\rangle_{s}^{\prime}=\left\langle f, v_{h}\right\rangle_{s} \text { for all } v_{h} \in-\bar{X}_{h} \text {. }
$$

Obviously, equations (2.1) are well defined if $s<m-n$ and $f \in H^{r}$ for $r>2 s-m$. As usual we consider the pseudodifferential operator $A$ as a bounded operator from $H^{s+n}$ in $H^{s-n}$ and write (2.1) as projection equations

$$
P_{s \div n, h}^{*} A u_{h}=P_{s \div n, h}^{*} f,
$$

where $P_{s ; n, h}^{*}$ denotes the formal $s$-adjoint operator of the orthogonal projection $P_{s+n, h}: H^{s+n} \rightarrow X_{h}$ defined by

$$
\left\langle P_{s+n, h} u, v_{h}\right\rangle_{s+n}=\left\langle u, v_{h}\right\rangle_{s+n}^{i} \text { for all } v_{h} \in X_{h}
$$

and

$$
\left\langle P_{s ; n, h}^{*} u, v\right\rangle_{s}=\left\langle u, P_{s+n, h}\right\rangle_{s} \text { for } u \in H^{s-n}, v \in H^{s+n}
$$

Hence, the Galerkin method in $I^{s}$ is the projection method $\left\{X_{h}, P_{s+n, h}^{*}\right\}$. We shall write $A \in \Pi\left(\left\{X_{h}, P_{8+n, h}^{*}\right\} ; H^{i}\right)$ if equations (2.2) are uniquely solvable for all sufficiently small $h$ and all $f \in H^{t-2 n}$ and if the sequence of solution $\left\{u_{h}\right\}$ converges in the norm of $H^{t}$ to an exact solution of (1.4) as $h \rightarrow 0$. 
Lemma 2.1: Assume $A$ coercive elliptic (1.8) and $\operatorname{dim} \operatorname{ker} A=0$. Then $A \in \Gamma\left(\left\{X_{h}, P_{s+n, h}^{*}\right\} ; H^{s+n}\right)$.

Proof: In light of the results in [3: Th. 9.2] and [8] it suffices to show that $A$ satisfies the inequality .

$$
\operatorname{Re}\langle A u, u\rangle_{s} \geqq c\|u\|_{s+n}^{2}+c_{1}\|u\|_{s+n-\varepsilon}^{2}
$$

for some constants $c>0, \varepsilon>0$ and $c_{1}$ and all $u \in C^{\infty}$. Let us define the mapping $A^{t}, t \in \mathbf{R} ;$ by

$$
\Lambda^{l} u(x)=\sum_{0 \neq k \in z} \hat{u}_{k^{\prime}}|2 \pi k|^{\ell} \mathrm{e}^{2 \pi i k x}+\hat{u}_{0} .
$$

Obviously, $A^{t}$ is a pseudodifferential operator with symbol $|\xi|^{t}$. Furthermore, $A^{t} \Lambda^{s}=\Lambda^{t+s}$ and $\langle u, v\rangle_{s} \doteq\left\langle\Lambda^{t} u, \Lambda^{t} v\right\rangle_{s-t}$ for all $s \in \mathbf{R}$ and $u, v \in C^{\infty}$. Hence, $\langle A u, u\rangle_{s}$ $=\left\langle\Lambda^{2 s} A u, u\right\rangle_{0}$. The psendodifferential operator $\Lambda^{2 s} A$ is of order $2(s+n)$ and has the principal symbol $|\xi|^{2 s} a_{2 n}(x, \xi)$. Because of $\operatorname{Re}|\xi|^{2 s} a_{2 n}(x, \xi)>0$ on $\mathbf{R} \times\{ \pm 1\}$ the Gàrding inequality [9] yields

$$
\operatorname{Re}\left\langle\Lambda^{2 s} A u, u\right\rangle_{0} \cdot=\operatorname{Re}\langle A u, u\rangle_{s} \geqq c\|u\|_{s: n}^{2}+\cdot c_{1}\|u\|_{s+n-\varepsilon}^{2}
$$

Next we consider the convergence of the $H^{s}$-Galerkin method in a range of Sobolev spaces. To this end we formulate a special case of a general convergence theorem of projection methods $\left\{X_{h}, K_{h}\right\}$ with uniformly bounded projections $K_{h}$.

Lemina 2.2 [13]: If $\operatorname{dim} X_{h}=\operatorname{dim} \operatorname{im} K_{h}$ and $\left\|K_{h} u-u\right\|_{t-2 n} \rightarrow 0$ as $h \rightarrow 0$ for all $u \in H^{t-2 n}$ then the following two conditions are equivalent:

(i) $A \in \Pi\left(\left\{X_{h}, K_{h}\right) ; H^{i}\right)$;

(ii) there exists $A^{-1} \in L\left(H^{t-2 n}, H^{t}\right)$ and the finitedimensional operators $\left.K_{h} A\right|_{x_{n}}$ are stable in $H^{t}$, i.e., there exist constants $c>0$ and $h_{0}>0$ such that

$$
\left\|K_{h} A v_{h}\right\|_{t-2 n} \geqq c\left\|v_{h}\right\|_{t} \text { for all } h<h_{0} \text { and } v_{h} \in X_{h} \text {. }
$$

If one of the conditions is satisfied then the approximate solutions $u_{h}$ converge to the exact solution $u$ with quasioptimal rate

$$
\left\|u-u_{h}\right\|_{t} \leqq c \inf _{v_{n} \in X_{n}}\left\|u-v_{h}\right\|_{t} .
$$

In order to use Lemma 2.2 we consider some properties of the projections $P_{s+n, h}$.

Lemma 2.3: Lèt the spaces $X_{h}$ satisfy assumptions A 1 and A 2. Then the projections $P_{s+n, h}$ are uniformly bounded in $H^{r}$ for $2(s+n)-m<r<m$ and

$$
\left\|u-P_{s+n, h} u\right\|_{t} \leqq c h^{r-t}\|u\|_{r}
$$

for $2(s+n)-m-1 / 2 \leqq t \leqq r \leqq m+1 / 2, \imath<m, 2(s+n)-m<r$ and for all $u \in H^{\tau}$.

Proof: We use an argument of Nitsche [10]. Let $s+n<r<m$ and $u \in H^{r}$. Choosing $u_{h}$ from A 1 and using A 2 we conclude

$$
\begin{aligned}
& \left\|u-P_{s+n, h} u\right\|_{r} \leqq\left\|u-u_{h}\right\|_{r}+\left\|u_{h}-P_{s+n, h} u\right\|_{r} \\
& \leqq c\left(\|u\|_{r}+h^{s+n-r}\left(\left\|u_{h}-u\right\|_{s+n}+\left\|u-P_{s+n, h} u\right\|_{s+n}\right)\right) \leqq c\|u\|_{r} .
\end{aligned}
$$

For $2(s+n)-m<r<s+n$ we have

$$
\left\|P_{s+n, h} u\right\|_{r}=\sup _{, w \neq 0} \frac{\left\langle P_{s+n, h} u, w\right\rangle_{s+n}}{\|w\|_{2(s+n)-r}}=\sup _{w \neq 0} \frac{\left\langle u, P_{s+n, h} w\right\rangle_{s+n}}{\|w\|_{2(s+n)-r}} \leqq c\|u\|_{r} .
$$


Thus, for $2(s+n)-m<t<m$ estimate (2.5) follows immediately from the uniform boundedness and from A 1 . For $2(s+n)-m-1 / 2 \leqq t \leqq 2(s+n)-m$ we obtain

$$
\begin{aligned}
& \left\|u-P_{s+n, h} u\right\|_{t}=\sup _{w \neq 0} \frac{\left\langle u-P_{s+n, h} u, w\right\rangle_{s+n}}{\|w\|_{2(s+n)-t}} \\
& =\sup _{w \neq 0} \inf _{w_{h} \in X_{h}} \frac{\left\langle u-P_{s+n, h} u, w-w_{h}\right\rangle_{s+n}}{\|w\|_{2(s+n)-t}} \leqq\left\|u-P_{s+n, h} u\right\|_{t_{1}} \\
& \times \sup _{t o \neq 0} \inf _{w_{n} \in X_{h}} \frac{\left\|w-w_{h}\right\|_{2(s+n)-t_{1}}}{\|w\|_{2(s+n)-t}} \leqq c h^{l_{1}-t}\left\|u-P_{s+n, h}{ }^{\prime} u\right\|_{t} \\
& \leqq c h^{r-t}\|u\|_{r}
\end{aligned}
$$

where $2(s+n)-m<t_{1}<m, t_{1} \leqq r$

By duality (1.3) and (2.4) we derive

Lemma 2.4: Let $X_{h}(0 .<h<1)$ satisfy assumptions. A 1 and A 2. Then the projections $P_{s+n, h}^{*}$ are uniformly bounded in $H^{r}$ for $2 s-m<r<m-2 n$ and for $2 s-m-1 / 2 \leqq t \leqq r \leqq m-2 n+1 / 2, t<m-2 m, r>2 s-m$ we have

$$
\left\|u-P_{s+n, h}^{*} u\right\|_{t} \leqq c h^{r-t}\|u\|_{r} \text { for all } u \in H_{!}^{\dot{\tau}} \text {. }
$$

We are now in position to prove

Theorem 2.1: Let $A \in \Pi\left(\left\{X_{h}, P_{s ; n, h}^{*}\right\} ; H_{1}^{s+n}\right)$ and $X_{h}(0<h<1)$ satisfy assumptions A 1 and A 2 . Then $A \in \Pi\left(\left\{X_{h} ; P_{s+n . h}^{*}\right\} ; H^{t}\right)$ for $2(s+n)-m<t<m$. Moreover, for $f \in H^{r-2 n}, 2(s+n)-m<r \leqq m+1 / 2$ the approximate solutions converge in the norm of $H^{i}, 2(s+n)-m-1 / 2 \leqq t<m$ and $t \leqq r$, with optimal order to the exact solution:

$$
\left\|u-u_{h}\right\|_{t} \leqq c h^{r-t}\|u\|_{r} \leqq c h^{r-t}\|f\|_{-2 n} .
$$

Proof: The main step is to show that the operators $\left.P_{s+n, h}^{*} A\right|_{x_{n}}$ are stable in $H^{t}$. Let $2(s+n) \div m-1 / 2 \leqq t<s+n$. Since $A \in L\left(H^{t}, H^{t-2 n}\right)$ is invertible we obtain error estimates in spaces of lower order by applying Nitsche's trick [15]. There exists $v \in H^{2(s+n)-t}$ with $\left\|A^{*} v\right\|_{2 s+t}=1$, where $A^{*} \in L\left(H^{2(s+n)-t}, H^{2 s-t}\right)$ is the formal $s$-adjoint of $A$, such that $\left\|u-u_{h}\right\|_{t}=\left\langle u-u_{h}, A^{*} v\right\rangle_{s}$. Hence

and, consequently, :

$$
\begin{aligned}
\cdot\left\|u-u_{h}\right\|_{l} & =\left\langle A\left(u-u_{h}\right), v\right\rangle_{s}=\inf _{v_{h} \in X_{h}}\left\langle A\left(u-u_{h}\right), v-v_{h}\right\rangle_{t} . \\
\therefore \quad & \leqq\left\|A\left(u-u_{h}\right)\right\|_{s-n} \inf _{v_{n} \in X_{h}}\left\|v-v_{h}\right\|_{s+n} \leqq c h^{s+n-t}\left\|u-u_{h}\right\|_{s+n} .
\end{aligned}
$$

$$
\left\|A^{-1} f-\left(\left.P_{s+n, h}^{*} A\right|_{X_{n}}\right)^{-1} P_{s+n, h}^{*} f\right\|_{t} \leqq c h^{s+n-t}\|f\|_{s-n}
$$

for all $f \in H^{s-n}$. We set $f=\varphi_{h} \in \operatorname{im} P_{s \div n, h}^{*}$ and remark that

$$
\begin{aligned}
&\left\|\varphi_{h}\right\|_{s-n}=\sup _{w \neq 0} \frac{\left\langle\varphi_{h}, w\right\rangle_{s}}{\|w\|_{s+n}}=\sup _{w \neq 0} \frac{\left\langle\varphi_{h}, P_{s+n, h} w\right\rangle s}{\|w\|_{s+n}} \\
&: \quad \leqq\left\|\varphi_{h}\right\|_{t-2 n} \sup _{w \neq 0} \frac{\left\|P_{s+n, h} w\right\|_{2(s+n)-t}}{\|w\|_{s+n}}
\end{aligned}
$$

for $2(s+n)-m<t \leqq s+n$. Applying the inverse property A 2 we obtain

$$
\left\|P_{s+n, h} w\right\|_{2(s+n)-t} \leqq c h^{t-i s+n)}\left\|P_{s+n, h} w\right\|_{s+n} \leqq c h^{t-(s+n)}\|w\|_{s+n}
$$


and, hence

$$
\left\|\varphi_{h}\right\|_{s-n} \leqq c h^{(t-2 n)-(s-n)}\left\|\varphi_{h}\right\|_{t-2 n} .
$$

The results of [4: Th. 4.1.3] imply' that the last inequality holds for all $t<s+n$. Hence,

$$
\left\|\left(A^{-1}-\left(\left.P_{s ; n, h}^{*} A\right|_{X_{n}}\right)^{-1} P_{s+n, h}^{*}\right) \varphi_{h}\right\|_{t} \leqq c\left\|\varphi_{h}\right\|_{t-2 n} .
$$

and, consequently,

$$
\left\|P_{s \div n, n}^{*} A v_{h}\right\|_{t-2 n} \geqq c\left\|v_{h}\right\|_{t} \text { for } 2(s+n)-m-1 / 2 \leqq t \leqq s+n .
$$

In order to prove the stability of the operators $\left.P_{s+n, h}^{*} A\right|_{X_{n}}$ for $s+n<t<-m$ we assume that the exact solution $z \in H^{t}$. Then, using (2.5) and A 2 we derive

$$
\begin{aligned}
\left\|u-\dot{u}_{h}\right\|_{l} & \leqq\left\|u-P_{s+n, h} u\right\|_{t}+\left\|P_{s+n, h} u-u_{h}\right\|_{t} \\
. & \leqq c\left(\|u\|_{\ell}+h^{\ell-(s+n)}\left(\left\|P_{s+n, h} u-u\right\|_{s+n}+\left\|u-u_{h}\right\|_{s+n}\right) \leqq c\|u\|_{t} .\right.
\end{aligned}
$$

Hence

$$
\left\|\left(A^{-1}-\left(\left.P_{s+n, h}^{*} A\right|_{X_{n}}\right)^{-1} P_{s+n, h}^{*}\right)^{\prime} f\right\|_{\ell^{\prime}} \leqq c\|f\|_{t-2 n}
$$

for all $f \in H^{t-2 n}$. Since due to Lemma $2.4 \mathrm{im} P_{s+n, h}^{*} \subset H^{t-2 n}$ for $t<m$ we have shown that the operators

$$
\left.P_{s+n, h}^{*} A\right|_{X_{n}}: H^{t} \rightarrow H^{t-2 n}, \quad 2(s+n)-m-1 / 2 \leqq t<m
$$

àre stable. In view of Lemma 2.2 this implies together with Lemma' 2.4 the first assertion and estimate (2.7) for $2(s+n)-m<t<m$. Using once more Nitsche's trick we obtain (2.7) for $2(s+n)-m-1 / 2 \leqq t \leqq 2(s+n)-m$, which completes the proof

\section{Convergence of the collocation method}

In this section we connect the nodal collocation using splines of degree $d$ with a Galerkin method in $H^{j}$, where $j=(d+1) / 2$. 'Thus, we can apply results of Section 2 in order to study the spline collocation.

First we introduce some mappings. By $S_{0}$ we denote the pseudodifferential operator with symbol $\xi /|\xi|$ defined by

$$
S_{0} u(s):=\sum_{k=0}^{\infty} \hat{u}_{k} \mathrm{e}^{2 \pi i k x}-\sum_{k=-\infty}^{-1} \hat{u}_{k} \mathrm{e}^{2 \pi i k x} .
$$

Obviously, $S_{0} \in L\left(H^{s}\right), s \in \mathbf{R}$, and $\left.S_{0}^{-1}=S_{0}^{1}\right)$. Further, we define onedimensional operators $J_{\text {. and }} J_{\Delta}$ by

$$
J u:=\int_{0}^{1} u(x) d x \text { and } J_{\Delta} u:=\sum_{k=1}^{n} u\left(x_{k}\right)\left(x_{k+1}-x_{k-1}\right) / 2 .
$$

The following theorem goes back to D. ARnold and W. WEnduand and is fundamental for our further' considerations.

1) It is well known [7] that $S_{0}$ represents the Cauchy singular operator (1.10) on the unit circle $\Gamma=\{z:|z|=1\}$. 
'Theorem 3.1: Let $u \in H^{s}$ for s $>1 / 2$. Then the following conditions are equivalent:

(i) $u\left(x_{k}\right)=0$ for $k=1, \ldots, n$;

(ii) $\left\langle\left(I-J+J_{\Delta}\right) u, v_{\Delta}\right\rangle_{j}=0$ for all odd $d \geqq 1$ and all $v_{\Delta} \in S_{d}(A)$;

(iii) $\left(\left(I-J+J_{\Delta}\right) u, S_{0} v_{\Delta}\right\rangle_{j}=0$ for all even $d \geqq 0$ and all $v_{\Lambda} \in S_{d}(\Delta)$.

$$
\text { Proof: (i) } \leftrightarrow \text { (ii) see [2], (i) } \leftarrow \text { (iii) see [13] }
$$

We sec that the collocation equations (1.7) can be written as equations using inner products of Sobolev spaces. Since $A \in L\left(H^{s+n}, H^{s-n}\right)$ the equations (1.7) are well defined for $s+n<d+1 / 2$ and $s-n>1 / 2$, which imply in particular relation $d>2 n$ between the order-of pseudodifferential operator $A$-and the degree of the splines. Setting $s=j=(d+1) / 2$ all requirements are fulfilled and we obtain from Theorem 3.1 that the collocation equations (1.7) are equivalent to

$$
\left\langle\left(I-J+\dot{J}_{\Delta}\right) A \dot{u}_{\Delta}, v_{\Delta}\right\rangle_{j}=\left\langle\left(I-J+J_{A}\right) f, v_{\Delta}\right\rangle_{i}
$$

for $u_{\Delta}, v_{\Lambda} \in S_{d}(\Delta)$ and odd $d>2 n$

and to

$$
\begin{aligned}
& \left\langle\left(I-J+J_{A}\right) A u_{A}, S_{0} v_{\Delta}\right\rangle_{j}=\left\langle\left(I-J+J_{\Delta}\right) f, S_{0} v_{\Delta}\right\rangle_{j} \\
& \text { for } u_{\Delta}, v_{\Delta} \in S_{d}(\Delta) \text { and even } d>2 n .
\end{aligned}
$$

We note the error estimate for the trapezoidal rule

$$
\left|\left(J-J_{A}\right) u\right| \leqq c h_{\Delta}^{s}\|u\|_{s}
$$

for all $u \subseteq H^{s}$ and all meshes $\Delta$ if $1 \leqq s \leqq 2$, and for all $\gamma$-quasiuniform meshes with fixed $\gamma>0$ if $1 / 2<s<1$ (see [6]). For arbitrary meshes the error estimate

$$
\left|\left(J-J_{\Delta}\right) u\right| \leqq c h_{A}^{s}\|u\|_{s}
$$

is easily established, where $0<\varepsilon<s-1 / 2$. 'Thus, one can expect that equations (1.7) are uniquely solvable for all sufficiently small $h_{\Delta}$ if for odd $d$ equations

$$
\left\langle A u_{\Delta}, v_{\Delta}\right\rangle_{i}=\left\langle f, v_{\Delta}\right\rangle_{j} \text { for all } v_{\Lambda} \in S_{d}(\Lambda)
$$

or if for even $d$ equations

$$
\left\langle A u_{\Delta}, S_{0} v_{\Delta}\right\rangle_{j}=\left\langle f, S_{0} v_{A}\right\rangle_{j} \text { for all } v_{\Delta} \in S_{d}(\Delta)
$$

are uniquely solvable.

We remark that (3.3) is the Galerkin method $\left\{S_{d}(\Delta), P_{j \div n, A}^{*}\right\}$ applied to operator $A$, where $P_{j+n: \Delta}$ is the orthogonal projection of $H^{j+n}$ onto $S_{d}(\Delta)$. Introducing $\tilde{S}_{d}(\Delta)$ $:=S_{0} S_{d}(\Lambda)$ and denoting by $\widetilde{P}_{j+n, \Delta}$ the orthogonal projection of $H^{j+n}$ onto $\widetilde{S}_{d}(\Delta)$ we see, that (3.4) is the Galerkin method $\left\{\tilde{S}_{d}(\Delta), \tilde{P}_{j+n, \Delta}^{*}\right\}$ applied to the operator $A S_{0}$.

In order to prove the hypothesis we shall write equations (1.7) as projection equa'tions using some interpolation projections $Q_{d, \Delta}$ with

$$
\operatorname{im} Q_{d, A}=\operatorname{im} P_{j+n, 4}^{*} \text { for odd } d,
$$

resp.

$$
\operatorname{im} Q_{d, \Delta}=\operatorname{im} \tilde{P}_{j+n, \Delta}^{*} \quad \text { for even } d .
$$

The existence of these interpolation projections follows from Theorem 3.1.

Let $d$ be odd. Then the interpolating, element $Q_{d, d} u \in \operatorname{im} P_{j \neq n . d}^{*}$ has to satisfy

$$
\left\langle\left(I-J+J_{\Delta}\right) Q_{d, \Delta} u, v_{\Delta}\right\rangle_{j}=\left\langle\left(I-J+J_{A}\right) u, v_{\Delta}\right\rangle_{j}
$$


for all $v_{\Delta} \in S_{d}(\Delta)$. Hence

$$
P_{j: n, \Delta}^{*}\left(I-J+J_{\Delta}\right) Q_{d, \Delta} u=P_{j ; n, \Delta}^{*}\left(I-J+J_{\Delta}\right) u
$$

for all $u^{i} \in H^{j-n}$. Since im $P_{j+n, 4}^{*}$ contains the constant functions and $\left(I=J+J_{\Delta}\right)^{-1}$ $=\left(I+J-J_{\Delta}\right)[2]$ we obtain

$$
\therefore Q_{d, \Delta}=\left(I+J-J_{\Delta}\right) P_{j+n, d}^{*}\left(I-J+J_{A}\right)=P_{j+n, \Delta}^{*}+\left(J-J_{\Delta}\right)\left(P_{j+n, d}^{*}-I\right) .
$$

Analogously one obtains for even $d$

$$
Q_{d, \Lambda}=\tilde{P}_{j+n, 4}^{*}+\left(J^{*}-J_{\Delta}\right) \cdot\left(\tilde{P}_{j+, n, 4}^{*}-I\right) .
$$

Thus, the nodal spline collocation for equation (1:4) is the projection method $\left\{S_{d}(\Delta), Q_{d, 4}\right\}$.

We are now in position to prove

Lemma 3.1: Let:the degree of the splines $d$ be odd. Then

$A \in I I\left(\left\{S_{d}(\Delta), Q_{d . \Delta}\right\} ; H^{j+n}\right)$ if and only if $A \in I I\left(\left\{S_{d}(\Delta), P_{j: n, d}^{*}\right\} ; H^{j+n}\right)$.

If dis even then

$$
A \in \Pi\left(\left\{S_{d}(\Delta), Q_{d, \Delta}\right\} ; H^{i+n}\right) \text { if and only if } A S_{0} \in I I\left(\left\{\tilde{S}_{d}(\Delta), \tilde{P}_{j+n, 4}^{*}\right\} ; H^{j+n}\right) .
$$

P'roof: Since the finitedimensional spaces $S_{d}(\Delta)$ and $\tilde{S}_{d}(\Delta)$;satisfy approximation property $A 1$ with $m=d+1 / 2$ and $h=-h_{\Delta}$ the projections $P_{j+n, 4}^{*}, \tilde{P}_{j: n, 4}^{*}$ and $Q_{d, \Delta}$ strongly converge, in $H^{i-n}$ to the identity operator. Thus, we can apply Lemma 2.2 and have to study the stability of the finitedimensiorial operators as $h_{\mathrm{d}} \rightarrow 0$.

Let $d$ be odd. Since $j-n>1 / 2$ we obtain from (3.5), (3.1) and (3.2) $\| P_{j ; n}^{*}$ $-Q_{d . \Delta} \|_{j-n} \rightarrow 0$, hence the operators $\left.Q_{d, A} A\right|_{S_{d(A)}}$ are stable in $H^{j+n}$ if and only if $\left.P_{j+n, 1}^{*} A\right|_{S_{d}(\Delta)}$ are stable. For even $d$ we derive from $(3.6),(3.1)$ and (3.2) $\| \tilde{P}_{j+n, 4}^{*}$ $-Q_{d, A} \|_{j-n} \rightarrow 0$ and hence, the operators $\left.Q_{d, \Delta} A\right|_{S_{d}(\Delta)}=Q_{d, \Delta} A S_{0}||_{s_{d}(\Delta)}$ are stable in $H^{j+n}$ if and only if $\left.\tilde{P}_{j+n . d}^{*} A S_{0}\right|_{\tilde{s}_{\mathfrak{d}}(\Delta)}$ are stable

We can now give conditions for the convergence of the spline collocation. We remark that in the case of odd degree $d$ this condition was already established in [2]. Here we give a different proof.

Theorem 3.2: If $\operatorname{dim} \operatorname{ker} A=0$ and for odd $d>2 n$ the operator $A$ is strongly elliptic or for even $d>2 n$ the operator, $A S i s$ strongly elliptic then $A \in I l\left(\left\{S_{d}(\Delta), Q_{d, d}\right\}\right.$; $\left.H^{i+n}\right)$. Furthermore, for $f \in H^{r-2 n}, j+n \leqq r \leqq d+1$, we have.

$$
\left\|u-u_{\Delta}\right\|_{j+n} \leqq c h_{\Delta}^{r-(j+n)}\|f\|_{r-2 n} \text {. }
$$

( $S$ denotes the Cauchy singular operator (1.10):)

Proof: By. 0 we denote the operator of multiplication with a nonzero function. $\vartheta(x) \in C^{\infty}$. Obviously, $Q_{d . \Delta} \theta^{-1} Q_{d,\lrcorner} 0 A=Q_{d, \Delta} A$ and $0^{-1} \in \Pi\left(\left\{\operatorname{im} Q_{d . \Delta}^{\prime}, Q_{d . \Delta}\right\} ; H^{j-n}\right)$. Hence, $A \in I I\left(\left\{S_{d}(\Delta), Q_{d . A}\right\} ; H^{i+n}\right)$ if and only if $0 A \in I I\left(\left\{S_{d}(\Delta), Q_{d . \Delta}\right\} ; H^{j+n}\right)$, and because of Temma 3.1, if and,only if

$$
0 A \in \Pi\left(\left\{S_{d}(\Delta), P_{j+n, \Delta}^{*}\right\} ; \quad H^{j+n}\right) \text { for odd } d
$$

for

$$
0 A S_{0} \in \Pi\left(\left\{\tilde{S}_{d}(\Delta), \mathscr{P}_{j+n, \Delta}^{*}\right\} ; H^{j+n}\right) \text { for even } d
$$


and some nonzero function $\vartheta$. Due to Lemma $\dot{2} .1$ (3.8) holds if $\operatorname{Re} \vartheta(x) \dot{a}_{2 n}(x, \xi)>0$ on $\mathbf{R} \times\{ \pm 1\}$, i.e., if $A$ is strongly elliptic. (3.9) is valid if $\operatorname{Re} \vartheta(x) \cdot a_{2 n}(x, \xi) \xi /|\xi|>0$ on $\mathbf{R} \times\{ \pm 1\}$; i.e., if the operator $A S$ is strongly elliptic. Estimate (3.7) follows from Lemma 2.2 and $A 1$

Let us derive equivalent conditions for the strong ellipticity of $A$ and $A S$. Since $a_{2 n}(x, \xi)$ is positively homogeneous of degree $2 n$ with respect to $\xi$, there holds

with

$$
\begin{aligned}
a_{2 n}(x, \xi) & =|\xi|^{2 n}\left(a_{2 n}(x,+1)(1+\xi /|\xi|) / 2+a_{2 n}(x,-1)(1-\xi /|\xi|) / 2\right) \\
& =|\xi|^{2 n}(c(x)+d(x) \xi /|\xi|)
\end{aligned}
$$

$$
c(x)^{\prime}=\frac{a_{2 n}(x,+1)+a_{2 n}(x,-1)}{2}, d(x) \doteq \frac{a_{2 n}(x,+1)-a_{2 n}(x,-1)}{2} .
$$

Hence, $\operatorname{Re} \vartheta(x) a_{2 n}(x, \xi)>0$ on $\mathbf{R} \times\{ \pm 1\}$ iff $\operatorname{Re} \vartheta(x)(c(x) \pm d(x))>0$ on $\mathbf{R}$. The - last relation holds for some function $\vartheta \in C^{\infty}$ if and only if $c(x)+\lambda d(x) \neq 0$ for $x \in \mathbf{R}, \lambda \in[-1,1]$ [11: Lemma 4.4]. Thus, we obtain that $A$ is strongly elliptic if and only if

$$
\mu a_{2 n}(x,+1)+(1-\mu) a_{2 n}(x,-1) \neq 0 . \text { for } \quad x \in \mathbf{R}, \mu \in[0,1] .
$$

Analogeously, operator $A S$ is strongly elliptic if and only if $d(x)+\lambda \cdot c(x) \neq 0$ for $x \in \mathbf{R}, \lambda \in[-1,1]$, or equivalently,

$$
\mu a_{2 n}(x,+1)-(1-\mu) a_{2 n}(x,-1) \neq 0 ; \text { for } \cdot \dot{x} \in \mathbf{R}, \mu \in[0,1] .
$$

We now investigate the convergence of the nodal spline collocation in a range of Sobolew spaces. To this end we remark that the spaces $S_{d}(\Delta)$ and $\tilde{S}_{d}(\Delta)$ satisfy the inverse property A 2 with $m=d+1 / 2$ and $h=h_{b}$ if all meshes under consideration are $\gamma$-quasiuniform with fixed $\gamma>0$ (cf. [6]).

Theorem 3.3: Suppose $\operatorname{dim} \operatorname{ker} A=0$ and $A \in \mathscr{D}_{\gamma}$. If $A$ satisfies (3.10) and $d$ is odd or if $A$ satisfies (3.11) and $d$ is even then $A \in \Pi\left(\left\{S_{d}(\Delta), Q_{d .4}\right\} ; H^{t}\right)$ for $2 n+1 / 2$ $<t<d+1 / 2$. Moreover, for any right-hand side $f \in H^{r-2 n}, 2 n+1 / 2<r \leqq d+1$, the approximate solutions $u_{d}$ converge in the norm of $H^{t}, 2 n \leqq t<d+1 / \overline{2}, t \leqq r$, to the exact solution $u$ with

$$
\left\|u-u_{\Delta}\right\|_{t} \leqq c h_{\Delta}^{r-t}\|f\|_{r-2 n} .
$$

Proof: Due to Lemma 2.4 the projections $P_{j+n, 4}^{*}$ and $\tilde{P}_{j+n, 4}^{*}$ are uniformly bounded in $H^{t-2 n}$ for $2 n+1 / 2<t<d+1 / 2$. Hence, using error estimate (3.1) and representations $(3.5)$, (3.6) we conclude that the interpolation projections $Q_{d, \Delta}$ strongly converge to the identity operator in $H^{t-2 n}$ as $h_{\Delta} \rightarrow 0$. Thus, the first assertion is proved if we show that the operators $\left.Q_{d, \Delta} A\right|_{S_{t}(A)}$ are stable in $H^{t}$.

Let $d$ be odd. Because of Theorem 3.2, Lemmas 2.2 and 3.1 condition (3.10) yields the stability of $\left.P_{j: n, A}^{*} A\right|_{\dot{S}_{\mathfrak{d}}(\Delta)}$ in $H^{j+n}$ as $h_{\Delta} \rightarrow 0$. The proof of Theorem 2.1 shows tháat.

$$
\left\|P_{j+n, \Delta}^{*} A v_{\Delta}\right\|_{t-2 n} \geqq \dot{c}\left\|v_{\Delta}\right\|_{t}
$$

for all $\gamma$-quasiuniform meshes $\Delta$ with $h_{\Delta}<h$, all $v_{d} \in S_{d}(\Delta)$ and $2 n \leqq t<d+1 / 2$. Formula (3.5) implies

$$
\left\|\left(Q_{d, \Delta}-P_{j+n, A}^{*}\right) v\right\|_{l-2 n}=j\left(J-J_{\Delta}\right)\left(P_{j+n, 4}^{*}-I\right) v \mid \leqq c h^{r}\|v\|_{r}
$$

for $v \in H^{r}, r>1 / 2$, such that

$$
\left\|Q_{d, \Delta}-P_{j+n, \Delta}^{*}\right\|_{t-2, n} \rightarrow 0 \text { for } 2 n+1 / 2<t<d+1 / 2,
$$


which proves the stability of $\left.Q_{d, \Delta} A\right|_{S_{d}(\Delta)}$ in $H^{t}$. If $d$ is even then condition (3.11) implies the stability of $\tilde{P}_{j+n}^{*} A S_{0} \mid \tilde{s}_{d}(\Delta)$ in $H^{t}$ for $\gamma$-quasiuniform meshes and $2 n \leqq t<d$ $+1 / 2$. (3.6) yields $\left\|Q_{d, \Delta}-\tilde{P}_{j+n, \Delta}^{*}\right\|_{\ell-2 n} \rightarrow 0$ for $2 n+1 / 2<t<d+1 / 2$ and, consequently, the stability of

$$
\left.Q_{d, \Delta} A S_{0}\right|_{\tilde{S}_{d}(\Delta)}=\left.Q_{d, \Delta} A\right|_{S_{\mathrm{d}}(\Delta)} \text { in } H^{t} .
$$

In view' of I.enma $2.2 A \in I I\left(\left\{S_{d}(\Delta), Q_{d, d}\right\} ; H^{t}\right)$ and estimate (3.12) holds for $2 n+1 / 2$ $<t<d+1 / 2$.

In order to establish (3.12) in the remaining case $2 n \leqq t \leqq 2 n+1 / 2$ we first consider the case of odd $d$ and remark that because of (3.5) $u_{\Delta}$ solves the equation

$$
P_{j+n, d}^{*} A u_{1}+\left(J-J_{\Delta}\right)\left(P_{j+n, d}^{*}-I\right) A u_{\Delta}=P_{j: n, \Delta}^{*} f-\left(J-J_{\Delta}\right)\left(P_{j+n}^{*}-I\right) f \text {. }
$$

Denoting by $u_{d}^{\prime}$ the solution of the Galerkin equation

$$
P_{j \neq n, \Delta}^{*} A u_{\Delta}^{\prime}=P_{j+n, \Delta}^{*}
$$

we obtain

$$
P_{j+n . \Delta}^{*} A\left(u_{\Delta} \div u_{\Delta}{ }^{\prime}\right)=\left(J-J_{\Delta}\right)\left(P_{j+n . \Delta}^{*}-I\right)^{\prime}\left(j-A u_{\Lambda}\right)
$$

Hence, using (3.13), (3.1) and Lemina 2.4 we derive ${ }^{i}$

$$
\begin{aligned}
\left\|u_{\Delta}-u_{\Delta}{ }^{\prime}\right\|_{t} & \leqq c\left\|P_{j \div n}^{*} A\left(u_{\Delta}-u_{\Delta}{ }^{\prime}\right)\right\|_{t-2 n} \leqq c h_{\Delta}^{r-2 n}\left\|A u_{\Delta}-f\right\|_{r-2 n} \\
& \leqq c h_{\Delta}^{r-2 n}\|f\|_{r-2 n} .
\end{aligned}
$$

Theorein 2.1 states that $\left\|u-u_{j^{\prime}}\right\|_{l} \leqq c h_{d}^{r-t} \cdot\|f\|_{r-2 n}$ and therefore

$$
\left\|u-u_{\Delta}\right\|_{t} \leqq c\left(h_{d}^{r-t}\|f\|_{r-2 n}+h_{\Delta}^{r-2 n}\|f\|_{r-2 n}\right) \leqq c h_{\Delta}^{r-t} \cdot\|f\|_{r-2 n} .
$$

The same arguments prove estimate (3.12) in the case of even $d$

\section{Convergence of the standard Galerkin method}

The example of the Galerkin method using trigonometrical polynomials, which converges for the operator of wultiplication with a nonzero function if and only if this function has the winding number zero [7], shows that in general Galerkin methods do not converge for strongly elliptic operators. In this section we shall demonstrati that the standard Galerkin procedure with splines converges for strongly elliptic pseudodifferential equations as it is stated without proof in some papers. Our proof utilizes essentially results of Section 3.

First we consider relations between Galerkin methods in different Sobolev spaces and using splines of different degree.

Let us denote by $D^{\prime}, l \in \mathbf{Z}$, the pseudodifferential operator

$$
D^{l} u=\sum_{0 \neq k \in Z} \hat{u}_{k}(2 \pi k)^{l} \mathrm{e}^{2 \pi i k x}+\hat{u}_{0}
$$

having the symbol $\xi^{l}$. Obviously, for sufficiently smooth $u$ and $l \in \mathbf{N}$ we have $D^{l} u=\left(\frac{1}{i} \frac{d}{d x}\right)^{l} u+J u$. Furthermore, $D^{l}$ maps $H^{s}(s \in \mathbf{R})$-isomorphically onto $H^{s-l}$ and $\left\|D^{l} u\right\|_{s-l}=\|u\|_{s}$ : Using the periodicity of the splines we obtain

$$
S_{d-l}(\Delta)=D^{l} S_{d}(\Delta) \text { for } l \leqq d .
$$

In difference to Chap. 3 the orthogonal projection of $H^{s}, s<d+1 / 2$, onto the subspaces $S_{d}(\Delta)$ is now denoted by $P_{s, \Delta, d}$. It is easily seen that

$$
P_{s-l, \Delta, d-l}=D^{l} P_{s, \Delta . d} D^{-l}, \quad l \leqq d .
$$


I.emma 4.1: Let $s+n<d+1 / 2$ and the integer $l \leqq d$. Then $A \in \Pi\left(\left\{S_{d-l}(\Delta)\right.\right.$, $\left.\left.P_{s-l+n, d, d-l}^{*}\right\} ; H^{s-1: n}\right)$ if and only if $A . \in \Pi\left(\left\{S_{d}(\Delta), P_{s ; n, d, d}^{* *}\right\} ; H^{s-n}\right)$.

Proof: It suffices to prove the assertion in one direction. Let $A \in I I\left(\left\{S_{d}(\Delta)\right.\right.$, $P_{s+n, A, d}^{*} ; H^{s+n)}$ and suppose that the operators $\left.P_{s-l+n, \Delta, d-l}^{*} A\right|_{S_{d-1}(\Delta)}$ are not stable in $H^{s-l+n}$ as $h_{\lrcorner} \rightarrow 0$. Then there exists a sequence $\left\{v_{\Delta} \in S_{d-l}(\Delta)\right\}$ with $\left\|v_{\Delta}\right\|_{s-l+n}=1$ weakly converging to some $v \in H^{s-1+n}$ such that

$$
\left\|P_{s-l+n, d, d-l}^{*} A v_{\Delta}\right\|_{s-l-n} \rightarrow 0 \text {. }
$$

Since $P_{s-t+n, \Delta, d-l}^{*} A v_{A}$ weakly converge to $A v$ in $H^{s-\imath-n}$ we obtain $v=0$.

Using (4.1) we get on the other hand

$$
P_{s-l+n, d, d-l}^{*} A v_{\Delta}=D^{l} P_{s+n, A, d}^{*} D^{-l} A v_{\Delta}=D^{\imath} P_{s+n, d, d}^{*} A D_{1}^{-l} v_{\Delta}+D^{l} P_{s+n, \Delta, d}^{*} K v_{A}
$$

where $K=D^{-l} A-A D^{-l}$ is a pseudodifferential operator of order $2 n-l-1$. Hence, $\left\|K v_{A}\right\|_{s-n} \rightarrow 0$. Because of

$$
\left\|P_{s-l+n, \Delta, d, l}^{*} A v_{\Delta}\right\|_{s-l-n}=\left\|P_{s+n, \Delta, d}^{*}\left(A D^{-l} v_{\Delta}+K v_{\Delta}\right)\right\|_{s-n}
$$

we derive $\left\|P_{s+n, A, d}^{*} A D^{-l} v_{d}\right\|_{s-n} \rightarrow 0$, which is impossible since $D^{-l} v_{d} \in S_{d}(\Delta)$ and $\left\|D^{-l} v_{\Delta}\right\|_{s+n} \doteq\left\|v_{1}\right\|_{s-l+n}=1$.

Theorem 4.1: Let the pseudodifferential operator $A$ be strongly elliptic and $\operatorname{dim} \operatorname{ker} A=0$. Then $A \in \Pi\left(\left\{S_{d}(\Delta), P_{n .4 . d}^{*}\right\} ; H^{n}\right)$ for any $d>n-1 / 2$. For $f \in H^{r-2 n}$, $n \leqq r \leqq d+1$, the approximate solutions $u_{s}$ converge in $H^{t}, 2 n-d-1 \leqq t \leqq n$ to the exacl solution with the rate

$$
\left\|u-u_{\Delta}\right\|_{t} \leqq c h_{\Delta}^{r^{2}-t}\|f\|_{r-2 n} .
$$

If $\Delta \in \mathscr{D}_{y}$ then estimate (4.2) holds. for $2 n-d-1 \leqq t \leqq r \leqq d+1, t<d+1 / 2$, $r>2 n-d-1 / 2$.

Proof: Setting in Lemma $4.1 s=0, l=-(d+1)$ we obtain that $A \in \Pi l\left(\left\{S_{d}(A)\right.\right.$. $\left.\left.P_{n . d . d}^{*}\right\} ; H^{n}\right)$ if $A \in I I\left(\left\{S_{2 d+1}(A), P_{d+1+n, d, 2 d+1}^{*}\right\} ; H^{d+1+n}\right)$. From Iemma 3.1 and Theorem 3.2 we know that the Galerkin method in $H^{d+1}$ with splines of arbitrary odd degree $2 d+1>2 n$ converges for strongly elliptic pscudodifferential operators. Estimate (4.2) follows from Theorem 2.1

Using Lemma 4.2 we can also show that in a special case the strong ellipticity is necessary for the convergence of the standard Galerkin method.

Let $A$ be the singular integral operator

$$
A u(x)=a(x) u(x)+b(x) \cdot S u(x)
$$

and suppose that $A \in I I\left(\left\{S_{0}(\Delta), P_{0, \Delta, 0}^{*}\right\} ; H^{0}\right)$, i.e., Galerkin's method with piecewise constant functions converges in $H^{0}=L^{2}$. Due to Lemmas 4.1 and 3.1 we obtain $A \in I I\left(\left\{S_{1}(\Delta), Q_{1, A}\right\} ; H^{1}\right)$. But [13: Th. 4.8] states that $A$ must be strongly elliptic.

We want to mention that the Galerkin method can converge for degenerate and, in particular, for not strongly elliptic operators if the subprincipal symbol $a_{2 n-1}^{\prime}(x, \xi)$ $=a_{2 n-1}^{\prime}(x, \xi)-(2 i)^{-1} \partial^{2} a_{2 n} / \partial x \partial \xi$ satisfies additional requirements. The convergence of Galerkin's method with splines for such operators was considered by ELSCHNER [5] and as a special case of the obtained results one can formulate:

Suppose that $A$ is elliptic with $\operatorname{dim} \operatorname{ker} A=0$, but not strongly elliptic and satisfies

$$
\begin{aligned}
& \operatorname{Re} a_{2 n}(x, \xi) \geqq 0 \text { on } \mathbf{R} \times\{ \pm 1\}, \\
& \operatorname{Re} a_{2 n}^{\prime}(x, \xi)>0 \cdot \text { on } \sum_{\operatorname{Re} a_{2 n}}:=\left\{(x, \pm 1): \operatorname{Re} a_{2 n}(x, \pm 1)=0\right\} .
\end{aligned}
$$


Then for $f \in H^{r-2 n}, n+1 / 2 \leqq r \leqq d+1, n<d$, the standard Galerkin equations are uniquely solvable and the approximate solutions $u_{A}$ converge in the norm of $H^{n-1 / 2}$ to the exact solution with

$$
\left\|u-u_{\Delta}\right\|_{n-1 / 2} \leqq c h_{\Delta}^{r-1-(n-1 / 2)}\|f\|_{r-2 n} .
$$

Consequently, the set of the right-hand sides, for which Galerkin's method converges, and the order of convergence is smaller than in the strongly elliptic case and this cannot be improved in general (cf. [5]).

Finally we remark that all statements remain valid for the more general case of systems of pseudodifferential equations of the same order on a system of mutually disjoint $C^{\infty}$-curves. We only mention the equivalent conditions of the strong ellipticity for $A$ and $A S$ (cf. [12]):

$$
\operatorname{det}\left(\mu a_{2 n}(x,+1)+(1-\mu) a_{2 n}(x,-1)\right) \neq 0
$$

and respectively

$$
\operatorname{det}\left(\mu a_{2 n}(x,+1)-(1-\mu) \dot{a_{2 n}}(x,-1)\right) \neq 0
$$

for $x \in \mathbf{R}, \mu \in[0,1]$, where the matrix function $a_{2 n}(x, \xi)$ is the principal symbol of the system of equations."

\section{REFERENCES}

[1] Агранович, М. С.: Спектральные свойства эллиптиеских псепопифферешцальных операторов на замкнутой кривой. Функ. анал. $13, \mathfrak{2} 4$ (1979), $5 \dot{4}-\tilde{56}$.

[2] ArNol.D, D., and W. WENDLAND: On the asymptotic convergence of collocation methods. Math. Comput.' 41 (1983), 349-381

[3] Auris, J.-P.: Approximation of elliptic boundary-value problems (Pure and applied mathematics: Vol. 26). Wiley-Interscience: New York 1972.

[4] BABUŠKA, I., and A. K. Aztz: Survey lectures on the mathematical foundation of the finite element methöd. In: 'The mathematical foundation of the finite clement method with applications to partial differential equations. Proc. Symp. Univ. Maryland June 26-30, 1972 (Erl.: A. K. Azrz). Academic Press: New York-London 1972.

[5] Elschiner, J.: A Galerkin method with finite elements for degenerate onedimensional pseudodifferential equations. Math. Nachr. 111 (1983), 111-126

[6] Elschiner, J., and G! Scryidt: On spline interpolation in periodic Sobolev spaces. Preprint P-Math-01/83. Inst. Math. AdW DDR: Berlin 1983.

[7] Gochuer, I. Z., und I. A. Fridmax: Faltungsgleichungen und Projektionsverfahren zu ihrer Lösung (Ubers. a. d. Russ.; Math. Lehrbücher und Monographien: Abt. 2 Bd. 36). Akademie-Verlag: Berlin 1974.

[8] HildebraxdT, S., and E. Wiexholtz: Constructive proofs of representation theorems in separable Hilbert space. Comm. pure and appl. math. XVII (1964), 369-373.

[9] Konn, J. J., and L. Nimensera: On the algebra of pseudo-differential operators. Comm. pure and appl. math. XVIII (1965), 269-303.

[10] Nitsche, J.: Zur Konvergenz von Näherungsverfahren bezüglich verschiedener Normen. Num. Mith. 1; (1970), 224-227.

[11] Prösswórf, S., and G. Scimid: A finite element collocation method for singular integral equations. Math. Nachr. 100 (1981), 33-60.

[12] Prössdorf, S., and G. Schmidt: A finite element collocation method for systems of singular integral equations. Preprint P-Math-26/81. Inst. Math. AdW DDR: Berlin 1981. 
[13] Schмidт, G.: On spline collocation for singular ịtegral equations. Math. Nachr. 111 (1983), 177-196.

[14] Stephan, E., and W. WeNdeand: Remarks to Galerkin and least squares methods with finite elements for general elliptic problems. In: Lecture Notes in Mathematics 564. Springer-Verlag: Berlin-Heidelberg-New York 1976, pp. 461-471.

[15] Strang, G., and G. Fix: An analysis of the finite element method. Prentice Hall: Englewood Cliffs 1973.

Manuskripteingang: 20. 0i. 1983.

\section{VERFASSER:}

Dr. Gunther Schimidt

Institut für Mathematik der Akademie der Wissenschaften der DDR

DDR - 1086 Berlin, Mohrenstr. 39, PF 1304 